\title{
Ethnomedicinal plants used for digestive system disorders by the Karen of northern Thailand
}

\author{
Kornkanok Tangjitman ${ }^{1}$, Chalobol Wongsawad ${ }^{1 *}$, Kaweesin Kamwong ${ }^{2}$, Treetip Sukkho ${ }^{1}$ and Chusie Trisonthi ${ }^{1}$
}

\begin{abstract}
Background: Digestive system disorders have a substantial effect on worldwide morbidity and mortality rates, including in Thailand, where the majority of the rural areas have a lack of proper sanitation and awareness about disease prevention. This has led to the prevalence of different types of digestive diseases. Karen people in Thailand still use medicinal plants as first aid remedies in treating these diseases. Therefore, this study aimed at documenting the plants used to cure and prevent different types of digestive system disorders by Karen people of Chiang Mai Province, northern Thailand.

Methods: Ethnomedicinal data were collected from six key informants and 172 non-specialist informants regarding their traditional knowledge of medicinal plants. Quantitative approaches were used to determine Use Value (UV), Informant Consensus Factor (ICF) and Fidelity Level (FL) values.

Results: The study revealed that 36 medicinal plant species belonging to 31 genera and 24 families were used to treat digestive system disorders. The most prevalent plant families were Zingiberaceae (6 species), Euphorbiaceae (4 species) and Fabaceae (4 species). Leaves were the most commonly used plant part accounting for $32.6 \%$ of the plants, followed by the bark (18.6\%). About $60 \%$ of the administrations were given orally by potion (60\%) and consumption as food was also indicated (14\%). The highest ICF values were recorded for carminative disorders, stomachaches, geographic tongue, constipation, appetite stimulants and food poisoning (1.00 each) indicating the best agreement among the informants knowledge of medicinal plants that were used to treat aliments in these categories. The highest fidelity level values were recorded for Punica granatum (100.00), Psidium guajava (95.45), and Gymnopetalum integrifolium (90.91) showing conformity of knowledge on species with the best healing potential.

Conclusion: Medicinal plants still play an important role among Karen culture. The present information on these medicinal plants, which have high UV and FL values, may serve as the baseline data to initiate further research for the discovery of new compounds and the biological activities of these potential plant remedies. Further research on these plants may provide some important clues for the development of new drugs for the treatment of digestive system diseases.
\end{abstract}

Keywords: Ethnobotany, Traditional knowledge, Gastrointestinal, Chiang Mai, Pharmacology, Toxicology

\section{Introduction}

There are a wide number of digestive system disorders, which impose a substantial influence on morbidity and mortality rates, worldwide. The World Health Organization (WHO) [1] reported that digestive system disorders, particularly diarrhea, was the fifth leading cause of global mortality, as approximately 100 million people died worldwide in 2012 from these types of disorders.

\footnotetext{
* Correspondence: cwongsawad@gmail.com

'Department of Biology, Faculty of Science, Chiang Mai University, Huaykaew Road, Chiang Mai 50200, Thailand

Full list of author information is available at the end of the article
}

Moreover, in South-East Asia, diarrhea has been the cause of $10 \%$ of deaths among children below the age of 5 years.

For the last couple of years, there has been a global trend in the renewal of interest in a traditional system of treatments. Ethnomedicinal plant studies have become of particular interest and have become increasingly more valuable in the development of health care and conservation programs in different parts of the world [2]. The WHO has recognized the role of traditional medicine in the primary health care system [3]. In developing 
countries, medicinal plants continue to be a main source of medication. It has been estimated that approximately $88 \%$ of the inhabitants of underdeveloped countries rely mainly on traditional medicine for their primary form of medicinal health care [4].

Thailand has a rich population of ethnic people who still maintain a traditional knowledge of medicinal plants that are used in the treatment of illnesses [5]. Studies of several ethnomedicinal plants have been carried out among several ethnic groups in Thailand. However, there has been no comprehensive study of the medicinal plants used to treat digestive system disorders in Thailand. Digestive system disorders were identified as the third highest cause of morbidity among Thai people in 2010 [6]. More than 1 million people have appealed to the public health system for the treatment of these diseases. Besides, Jansongduang et al. [7] revealed that people who typically reside in remote areas especially hill tribe people in northern Thailand likely drink water from forest streams without any antiseptics and used the same water for bathing, raising livestock, and sewage disposal. These practices result in poor water quality and often lead to digestive system problems. Moreover, previous ethnobotanical studies in northern Thailand showed that digestive diseases had the highest number medicinal plants recorded compared with other illness categories and most hill tribe people had experience curing these diseases with medicinal plants [7-10]. This reflects that digestive system disorders are also important morbidity among Thai hill tribe people.

This study documents the traditional medicinal plants that are used for digestive system disorders by the Karen which comprise the largest hill tribe in Thailand [11]. The Karen originated in Tibet and had migrated to other parts of Southeast Asia, particularly Myanmar [12]. From the 18th century onwards they began to cross the Salween River and moved into Thailand, where they settled in the high mountains of Chiang Mai, Mae Hong Son and Lamphun provinces, as well as other areas. In 2003, the Karen people constituted $48 \%$ of the total hill tribe population in the region with a population more than 430,000 Karens in Thailand [11]. As they typically reside in the mountain areas, the Karen people have limited access to public healthcare systems. They have therefore accumulated a rich experience related to preventing and treating diseases with herbal remedies, and they have developed a distinctive knowledge of traditional medicine. This traditional knowledge has been handed down from one generation to the next by spoken word and through lifestyle. Most Karen villagers still maintain traditional knowledge of medicinal plants that are used for first aid remedies and to treat simple ailments [10].

\section{Materials and methods}

\section{Study area}

Data were collected in Chiang Mai province, northern Thailand (Figure 1). Chiang Mai province is surrounded by high mountain ranges and covers an area of approximately 20,107 $\mathrm{km}^{2}$ [13]. Forest area covers $17,640 \mathrm{~km}^{2}$ (72.01\%) of Chiang Mai's total area. The major types of forests in Chiang Mai are mixed deciduous forests, tropical evergreen forests and dry dipterocarp forests. Several national parks are also located in the province (Doi Inthanon, Doi Suthep-Pui, Mae Ping, Sri Lanna, Huay Nam Dang, Mae Phang and Chiang Dao). Six Karen villages (Huay Hea, Mai Lan Kam, Kew Pong, San Muang, Mai Sa Wan and Huay Pu Ling) were selected as study sites. These villages are located at 746, 692, $1,010,1,050,1,190$ and 1,050 m.l.s., respectively and they are surrounded by natural forests. There are 14 households in Huay Hea, 45 in Mai Lan Kam, 49 in Kew Pong, 51 in San Muang, 18 Mai Sa Wan and 32 in Huay Pu Ling. They typically consume upland rice and supplement their meals with vegetables and animal products for their diet. The villagers derive their main monetary income through the sale of forest products, livestock and as labor in northern Thai fields. Their economic status is generally considered to be poor. The Karen society is matriarchal. Each household contains only one or two generations. Most Karen people in Kew Pong, San Muang, Mai Sa Wan and Huay Pu Ling are Christian whereas those of Huay Hea and Mai Lan Kam consider themselves Buddhist.

\section{Data collection}

To collect plants and associated ethnomedicinal information relating to digestive system disorders from the Karen, field trips were conducted between 2006 and 2011. Initial contacts were made to the village headmen, to whom we explained the purpose and techniques of the proposed research. Subsequently the headmen explained the purpose and methods of the study to the villagers who gave their informed consent for the publication of this report and any accompanying images. The information on medicinal plants was gathered through interviews, guided tours, and participative observation in homegardens, cultivated fields and nearby forests. The plants used were indentified (local name), photographed and samples were collected for the preparation of herbarium specimens, which were deposited at the Faculty of Science, Chiang Mai University and the Queen Sirikit Botanical Garden Herbarium (QBG), Chiang Mai, Thailand. Plant identification was based largely on taxonomic literature, such as through the use of references entitled the Flora of Thailand, the Flora of China and the Flora of Java. 


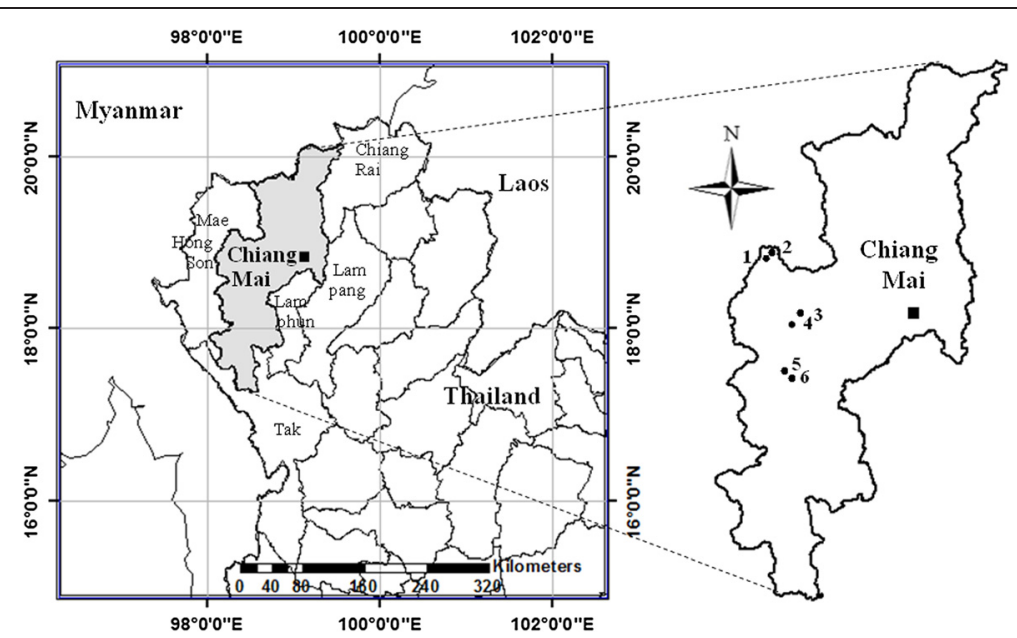

Figure 1 Map of study areas in Chiang Mai province, Thailand: (1) Kew Pong; (2) San Muang; (3) Mai Lan Kam; (4) Huay Hea; (5) Huay Pu Ling; (6) Mai Sa Wan.

Semi-structured interviews were conducted to determine the overall prevalence of medicinal plant knowledge. This was done from October 2011 through April 2012 with 172 non-specialist informants (80 males and 92 females, aged 13-92 years). All medicinal plant species data obtained from the key informant were prepared for the interview. During the interview, plant pictures and the Karen plant names were shown to the informant. Questions were asked individually concerning the actual use of the medicinal plants used in the therapy of digestive system disorders, along with questions about what plant part was used, the mode of preparation, and the route of administration. The semi-structured interviews were conducted in Thai in the presence of a translator when the informants were not able to communicate in the Thai language.

\section{Data analysis}

Use value (UV)

The relative importance was calculated employing the use value [14], a quantitative measure for the relative importance of a given species known locally:

$$
\mathrm{UV}=\sum U / \mathrm{n}
$$

where $U$ is the number of use-reports cited by each informant for a given species and $\mathrm{n}$ refers to the total number of informants. Use values are high when there are many use-reports for a plant, implying that the plant is important, and the approach is zero (0) when there are few reports related to its use. The use value, however, does not distinguish whether a plant is used for single or multiple purposes.

\section{Informant consensus factor (ICF)}

To test homogeneity of knowledge, the informant consensus factor was used [15]:

$$
\mathrm{ICF}=\left(N_{\mathrm{ur}}-N_{\mathrm{t}}\right) /\left(N_{\mathrm{ur}}-1\right)
$$

where $N_{\text {ur }}$ refers to the number of use-reports for a particular use category and $N_{\mathrm{t}}$ refers to the number of taxa used for a particular use category by all informants. A lower ICF value (near 0) indicates the informants' disagreement of using a particular plant to treat a particular ailment category, and a higher ICF value (approach 1 ) is indicative of using relatively few plants by the informants in the treatment of a particular ailment category [16].

\section{Fidelity level (FL)}

Because many plant species may be used in the same use category, it is interesting to determine the most preferred species used in the treatment of a particular ailment, which can be done with the Fidelity Level (FL) of Friedman et al. [17]:

$$
\mathrm{FL}(\%)=(N p / N) * 100
$$

where $N p$ is the number of use-reports cited for a given species for a particular use category and $N$ is the total number of use-reports cited for any given species. High FLs (near 100\%) are obtained for plants for which almost all use reports refer to the same way of using a given plant whereas low FLs are obtained for plants that are used for many different purposes.

\section{Results}

Medicinal plants and traditional uses

A total of 36 plants belonging to 31 genera and 24 families were recorded as being used by the Karen in 
treating different types of digestive system disorders (Table 1). The reported plant families include Zingiberaceae (6 species), Euphorbiaceae (4 species), Fabaceae (4 species), Musaceae (2 species), Acanthaceae, Apiaceae, Acoraceae, Asparagaceae, Celastraceae, Cucurbitaceae, Dilleniaceae, Flacourtiaceae, Juglandaceae, Lamiaceae, Lauraceae, Leeaceae, Melastomataceae, Myrsinaceae, Myrtaceae, Ochnaceae, Fabaceae, Poaceae, Punicaceae, Rhamnaceae and Rubiaceae (1 species each).

\section{Plant part used, method of preparation, and route of administration}

Among the different plant parts used - the leaf (32.6\%) was the most frequently used plant part, followed by the bark $(18.6 \%)$, the rhizome $(16.3 \%)$, fruit $(9.3 \%)$, the root $(7.0 \%)$, the stem $(7.0 \%)$, the whole plant $(4.7 \%)$, the flower $(2.3 \%)$ and the latex (2.3\%). Herbal medicines were prepared in the form of decoction (83.8\%), as well as those that were described as non-prepared (16.2\%). The modes of administration were potion (60.0\%), bath (18.0\%), eaten as food (14.0\%), held in mouth $(6.0 \%)$ and applied as liniment $(2.0 \%)$.

\section{Use records and aliments}

A total 902 actual use records were registered among the interview with non-specialist informants. These belong to 14 different aliments (Table 2). The ailment for which there was the most frequently reported was diarrhea, which accounted for slightly more than $23 \%$ of all use records while flatulence (22\%), laxative (19\%), gastric ulcer (19\%) and jaundice (10\%) were also common.

\section{Plant use values, informant consensus factor and fidelity levels}

The High Use Value was recorded for selected species, such as Zingiber ottensii (0.74), Zingiber montanum (0.72), Punica granatum (0.71), Psidium guajava (0.61), Senna occidentalis (0.59), Curcuma longa (0.56) and Dillenia pentagyna (0.52) (Table 1). High UV levels indicate high numbers of use reports by the informants for a particular plant.

The informant's consensus factor (ICF) was found to range between 0.00 and 1.00 (Table 2). The disease categories with the highest use reports were for carminative disorders, stomachaches, geographic tongue, constipation, as an appetite stimulant and for food poisoning (1.00 each), followed by flatulence and as a laxative (0.97 each), for diarrhea and mouth ulcers (0.95 each), gastric ulcers (0.92), jaundice (0.89), toothaches (0.50) and hemorrhoids (0.00).

The highest Fidelity Level (FL) for the plants used by the Karen was recorded as Punica granatum (100.00), followed by Psidium guajava (95.45), Gymnopetalum integrifolium (90.91), Zingiber montanum (90.20), Senna occidentalis (87.18), Dillenia pentagyna (84.62), Zingiber ottensii (81.75), Musa sapientum (81.58), Engelhardtia spicata var. colebrookeana (81.25), Melastoma malabathricum (76.92), Dendrocalamus strictus (75.00), Euphorbia heterophylla (72.22), Curcuma longa (70.63) and Senna alata (70.59) (Table 3).

\section{Toxicity of species used medicinally}

Toxicity data was available for only 18 of the 36 species. Table 4 shows general toxicity studies that have been previously recorded in evaluating the biological activities of these plants. Twelve species were reported to have a toxic effect on animals (Acorus calamus, Cassytha filiformis, Celastrus paniculatus, Euphorbia heterophylla, Euphorbia hirta, Flemingia macrophylla, Kaempferia parviflora, Senna alata, Senna occidentalis, Thunbergia laurifolia and Zingiber montanum), while six plants (Centella asiatica, Curcuma longa, Gmelina arborea, Psidium guajava, Punica granatum and Tamarindus indica) were reported to have no toxic effects on humans or animals.

\section{Discussion}

\section{Medicinal plant use}

The uses of 36 plant species used to treat 14 different aliments, by key and non-specialist informants, were reported in this study. The plants used most prevalently were from the family Zingiberaceae, which had six species. The Karen people prefer cultivating the Zingiberaceae plant for their personal consumption in their homegardens or in their fieldwork. Some of the most common dishes consumed by the Karen involve chili sauces and curry. The plants used in these dishes are usually cooked and the vegetables are sometimes part of the side dishes. Therefore, most Karen people are familiar with these plants and use them as food as well as for medicinal purposes.

As measured by the number of plant(s) per aliment, diarrhea was correlated with the highest number of plant species recorded (12 species) and also the highest number of uses recorded (209 uses). This might reflect the fact that diarrhea is quite prevalent in terms of morbidity among the Karen people. Most Karen villages are located in mountainous areas, which are geographically remote areas. Unsafe water supplies and inadequate levels of sanitation and hygiene may increase the transmission of diarrhea among the Karen people.

Leaves were the plant part that was used the most in the preparation of remedies by the Karen people, as compared to other parts. Many studies conducted elsewhere in northern Thailand also showed the dominance of the leaf in the preparation of remedies $[8,9,18]$. Leaves are the main photosynthetic organ in plants and are considered to be a key component of the natural pharmacy 
Table 1 Medicinal plants used to treat digestive system disorders by the Karen people of northern Thailand

\begin{tabular}{|c|c|c|c|c|c|c|c|}
\hline Scientific name (Voucher no.) & Family & Karen name & Part used & Preparation & Route of administration & Application & Use value \\
\hline Acorus calamus L. (K. Kamwong106) & Acoraceae & Por bue lah & rhizome & non, decoction & eaten as food, potion & stomach ache & 0.14 \\
\hline Asparagus filicinus Buch.-Ham. ex D.Don (K. Kamwong097) & Asparagaceae & Ya su mae & root & decoction & potion, bath & $\begin{array}{l}\text { gastric ulcer, jaundice hemorrhoid, } \\
\text { flatulence }\end{array}$ & 0.10 \\
\hline Boesenbergia rotunda (L.) Mansf. (K. Kamwong099) & Zingiberaceae & Por sa raw & rhizome & decoction & potion & flatulence & 0.39 \\
\hline Cassytha filiformis L. (K. Kamwong073) & Lauraceae & Se kruy po & all & decoction & potion, bath & jaundice & 0.21 \\
\hline Celastrus paniculatus Willd. (K. Kamwong031) & Celastraceae & Ti si bler & bark & decoction & potion & diarrhea & 0.08 \\
\hline Centella asiatica (L.) Urb. (T. Sukkho157) & Apiaceae & $\begin{array}{l}\text { Chuy po co la } \\
\text { do }\end{array}$ & leaf & non & eaten as food & gastric ulcer, diarrhea & 0.09 \\
\hline Croton kongensis Gagnep. (K. Kamwong032) & Euphorbiaceae & Sa ko wa & leaf & decoction & potion, bath & gastric ulcer, jaundice, diarrhea & 0.30 \\
\hline Croton robustus Kurz (K. Kamwong033) & Euphorbiaceae & Sa ko wa sui & leaf, bark & decoction & bath & jaundice & 0.21 \\
\hline Curcuma longa L. (T. Sukkho071) & Zingiberaceae & Si yaw & rhizome & non, decoction & eaten as food, potion & gastric ulcer, flatulence & 0.56 \\
\hline Dendrocalamus strictus (Roxb.) Nees (K. Kamwong284) & Poaceae & Wa mee & leaf & decoction & potion, bath & jaundice & 0.36 \\
\hline Dillenia pentagyna Roxb. (K. Kamwong204) & Dilleniaceae & Kho tee & bark & decoction & potion & gastric ulcer & 0.52 \\
\hline Embelia sessiliflora Kurz (T. Sukkho006) & Myrsinaceae & Blea blor & fruit & non & eaten as food & laxative & 0.09 \\
\hline $\begin{array}{l}\text { Engelhardtia spicata Blume var. colebrookeana } \\
\text { (Lindl. ex Wall.) Kuntze (K. Kamwong208) }\end{array}$ & Juglandaceae & Klue por & bark & decoction & potion & gastric ulcer & 0.59 \\
\hline Ensete glaucum (Roxb.) Cheesman (K. Kamwong020) & Musaceae & Ya pa la & leaf sheaf & decoction & potion & diarrhea, food poisoning & 0.23 \\
\hline Euphorbia heterophylla L. (T. Sukkho055) & Euphorbiaceae & Nor bo lo bell & leaf, latex & decoction, non & potion, liniment & laxative, mouth ulcer & 0.47 \\
\hline Euphorbia hirta L. (K. Kamwong272) & Euphorbiaceae & - & all & decoction & potion & gastric ulcer & 0.43 \\
\hline Flacourtia jangomas (Lour.) Raeusch. (K.Kamwong241) & Flacourtiaceae & Ser pae & bark & non, decoction & hold in mouth, potion & toothache, gastric ulcer, diarrhea & 0.14 \\
\hline Flemingia macrophylla (Willd.) Merr. (K. Kamwong209) & Fabaceae & Chor ae go bor & bark & decoction & potion & jaundice & 0.28 \\
\hline Gmelina arborea Roxb. (K. Kamwong122) & Lamiaceae & Ker ma & $\begin{array}{l}\text { bark, } \\
\text { flower }\end{array}$ & decoction & potion & gastric ulcer, laxative & 0.11 \\
\hline Gymnopetalum integrifolium Kurz. (T. Sukkho166) & Cucurbitaceae & $\begin{array}{l}\text { Se do kwaw } \\
\text { mee }\end{array}$ & leaf, stem & decoction & potion, bath & jaundice, flatulence & 0.19 \\
\hline Kaempferia parviflora Wall. ex Baker (T. Sukkho039) & Zingiberaceae & Por sue & rhizome & decoction & potion & gastric ulcer, flatulence & 0.17 \\
\hline Leea indica (Burm. f.) Merr. (K. Kamwong135) & Leeaceae & Na tor kor & root, stem & decoction & potion & diarrhea, hemorrhoid, gastric ulcer & 0.14 \\
\hline Melastoma malabathricum L. (K.Tang054) & Melastomataceae & Se la play & fruit & non & hold in mouth & mouth ulcer, geographic tongue & 0.37 \\
\hline Musa sapientum L. (K. Kamwong021) & Musaceae & Si kuy & fruit & non & eaten as food & diarrhea & 0.34 \\
\hline Mussaenda sanderiana Ridl. (K. Kamwong034) & Rubiaceae & Por jor kaw pe & root, leaf & decoction & $\begin{array}{l}\text { hold in mouth bath, } \\
\text { potion }\end{array}$ & $\begin{array}{l}\text { toothache, jaundice, appetite } \\
\text { stimulant }\end{array}$ & 0.17 \\
\hline Ochna integerrima (Lour.) Merr. (K. Kamwong028) & Ochnaceae & Ti si bor & leaf & decoction & potion, bath & $\begin{array}{l}\text { constipation, jaundice, gastric } \\
\text { ulcer, diarrhea }\end{array}$ & 0.25 \\
\hline Psidium guajava L. (K. Kamwong115) & Myrtaceae & Ma kwuy & $\begin{array}{l}\text { young } \\
\text { leaf }\end{array}$ & decoction & potion & diarrhea & 0.61 \\
\hline
\end{tabular}


Table 1 Medicinal plants used to treat digestive system disorders by the Karen people of northern Thailand (Continued)

\begin{tabular}{|c|c|c|c|c|c|c|c|}
\hline Punica granatum L. (T. Sukkho148) & Punicaceae & Chor pa lea & $\begin{array}{l}\text { young } \\
\text { leaf }\end{array}$ & decoction & potion & diarrhea & 0.71 \\
\hline Senna alata (L.) Roxb. (K. Kamwong168) & Fabaceae & $\begin{array}{l}\text { Ya la mer pa } \\
\text { do }\end{array}$ & leaf & decoction & potion & laxative & 0.49 \\
\hline Senna occidentalis (L.) Link (K. Kamwong183) & Fabaceae & Ya la mer & leaf & decoction & potion & laxative & 0.59 \\
\hline Tamarindus indica L. (K. Kamwong093) & Fabaceae & Sa mor klae & fruit & non & eaten as food & laxative & 0.26 \\
\hline Thunbergia laurifolia Lindl. (K. Kamwong083) & Acanthaceae & Jaw law lee der & leaf, stem & decoction & potion, bath & gastric ulcer diarrhea, jaundice & 0.16 \\
\hline $\begin{array}{l}\text { Zingiber montanum (J.König) Link ex A.Dietr. (K. } \\
\text { Kamwong236) }\end{array}$ & Zingiberaceae & Blae ko bor & rhizome & decoction, non & potion, eaten as food & flatulence, gastric ulcer & 0.72 \\
\hline Zingiber officinale Roscoe (K. Kamwong319) & Zingiberaceae & Sa ae & rhizome & decoction & potion & diarrhea, flatulence, gastric ulcer & 0.30 \\
\hline Zingiber ottensii Valeton (K. Kamwong166) & Zingiberaceae & Blae ko sue & rhizome & decoction & potion & flatulence, carminative & 0.74 \\
\hline Ziziphus cambodiana Pierre (K. Kamwong064) & Rhamnaceae & Ri co mae & bark & decoction & potion, bath & $\begin{array}{l}\text { gastric ulcer, hemorrhoid, } \\
\text { jaundice }\end{array}$ & 0.16 \\
\hline
\end{tabular}


Table 2 Category of digestive system disorders and their Informant Consensus Factor (ICF)

\begin{tabular}{llll}
\hline $\begin{array}{l}\text { Category of digestive } \\
\text { system disorder }\end{array}$ & $\begin{array}{l}\text { Number of use } \\
\text { reports }(\mathbf{N r})\end{array}$ & $\begin{array}{l}\text { Number of } \\
\text { taxa }(\mathbf{N t})\end{array}$ & ICF \\
\hline Diarrhea & 209 & 12 & 0.95 \\
Flatulence & 201 & 8 & 0.97 \\
Laxative & 171 & 6 & 0.97 \\
Gastric ulcer & 168 & 14 & 0.92 \\
Jaundice & 92 & 11 & 0.89 \\
Mouth ulcer & 21 & 2 & 0.95 \\
Geographic tongue & 6 & 1 & 1.00 \\
Constipation & 2 & 1 & 1.00 \\
Stomachache & 7 & 1 & 1.00 \\
Appetite stimulant & 2 & 1 & 1.00 \\
Food poisoning & 2 & 1 & 1.00 \\
Carminative & 15 & 1 & 1.00 \\
Toothache & 3 & 2 & 0.50 \\
Hemorrhoid & 3 & 3 & 0.00 \\
\hline
\end{tabular}

Table 3 Fidelity Level (FL) values for medicinal plants used by the Karen

\begin{tabular}{|c|c|}
\hline Category & $\begin{array}{l}\text { Most preferred species used against digestive } \\
\text { disorders (FL(\%)) }\end{array}$ \\
\hline Diarrhea & $\begin{array}{l}\text { Punica granatum (100.00), Psidium guajava (95.45), } \\
\text { Musa sapientum (81.58), Leea indica (50.44), Ensete } \\
\text { glaucum (50.00), Celastrus paniculatus (46.15), } \\
\text { Ochna integerrima (33.33) }\end{array}$ \\
\hline Gastric ulcer & $\begin{array}{l}\text { Dillenia pentagyna (84.62), Engelhardtia spicata var. } \\
\text { colebrookeana (81.25), Curcuma longa (70.63), } \\
\text { Croton kongensis (42.31), Ziziphus cambodiana } \\
\text { (36.36) }\end{array}$ \\
\hline Flatulence & $\begin{array}{l}\text { Zingiber montanum (90.20), Zingiber ottensii } \\
\text { (81.75), Boesenbergia rotunda (65.00), Kaempferia } \\
\text { parviflora (31.82) }\end{array}$ \\
\hline Laxative & $\begin{array}{l}\text { Senna occidentalis (87.18), Euphorbia heterophylla } \\
\text { (72.22), Senna alata (70.59), Tamarindus indica } \\
\text { (62.75) }\end{array}$ \\
\hline Jaundice & $\begin{array}{l}\text { Gymnopetalum integrifolium (90.91), Dendrocalamus } \\
\text { strictus (75.00), Croton robustus (56.25), Flemingia } \\
\text { macrophylla (46.15), Mussaenda sanderiana (44.19) }\end{array}$ \\
\hline Mouth ulcer & Melastoma malabathricum (76.92) \\
\hline Geographic tongue & Melastoma malabathricum (23.08) \\
\hline Constipation & Ochna integerrima (2.38) \\
\hline Stomachache & Acorus calamus (6.09) \\
\hline Appetite stimulant & Mussaenda sanderiana (4.65) \\
\hline Food poisoning & Ensete glaucum (7.14) \\
\hline Carminative & Zingiber ottensii (10.95) \\
\hline Toothache & Mussaenda sanderiana (4.65) \\
\hline Hemorrhoid & Ziziphus cambodiana (9.09) \\
\hline
\end{tabular}

Table 4 Literatures reporting on toxicity studies for medicinal plants used by the Karen

\begin{tabular}{|c|c|}
\hline Scientific name & Toxic effect \\
\hline Acorus calamus $L$. & $\begin{array}{l}\text { acute toxicity in mice, } L D_{50}=221 \mathrm{~g} / \mathrm{kg} \\
{[44]}\end{array}$ \\
\hline Cassytha filiformis L. & $\begin{array}{l}\text { acute toxicity in mice, } L_{50}=625.8 \mathrm{~g} / \mathrm{kg} \\
{[45]}\end{array}$ \\
\hline Celastrus paniculatus Willd. & $\begin{array}{l}\text { hyperactivity and loss of righting reflex } \\
\text { in rat }[46]\end{array}$ \\
\hline Euphorbia heterophylla L. & increase leucopaenia in rat [47] \\
\hline Euphorbia hirta L. & $\begin{array}{l}\text { leucocytosis, dullness, anorexia, stairy } \\
\text { haircoat and } 20 \% \text { mortality in rat [47] }\end{array}$ \\
\hline $\begin{array}{l}\text { Flemingia macrophylla } \\
\text { (Willd.) Merr. }\end{array}$ & $\begin{array}{l}\text { severe hypoglycemia followed by death } \\
\text { within } 24 \text { hour after administration to } \\
\text { mice [48] }\end{array}$ \\
\hline $\begin{array}{l}\text { Kaempferia parviflora Wall. } \\
\text { ex Baker }\end{array}$ & hepatotoxic to rat [49] \\
\hline Senna alata (L.) Roxb. & $\begin{array}{l}\text { decrease hemoglobin and erythrocyte } \\
\text { (RBC) count values in rats [36] }\end{array}$ \\
\hline Senna occidentalis (L.) Link & $\begin{array}{l}\text { intestinal disturbance in long-term used } \\
\text { in rats [50] }\end{array}$ \\
\hline Thunbergia laurifolia Lindl. & $\begin{array}{l}\text { decrease red blood cell in male mice } \\
\text { [51] }\end{array}$ \\
\hline $\begin{array}{l}\text { Zingiber montanum } \\
\text { (J.König) Link ex A.Dietr. }\end{array}$ & $\begin{array}{l}\text { acute toxicity in mice, } \mathrm{LD}_{50}=80 \mathrm{~g} / \mathrm{kg} \\
{[52]}\end{array}$ \\
\hline Zingiber officinale Roscoe & embryo toxic to pregnant rats [53] \\
\hline Centella asiatica (L.) Urb. & no toxic effect in mice [54] \\
\hline Curcuma longa $\mathrm{L}$. & no toxic effect in human [55] \\
\hline Gmelina arborea Roxb. & no toxic effect in rodents [56] \\
\hline Psidium guajava $\mathrm{L}$. & no toxic effect in mice [57] \\
\hline Punica granatum $\mathrm{L}$. & no toxic effect in rats [58] \\
\hline Tamarindus indica $\mathrm{L}$. & no toxic effect in mice [59] \\
\hline
\end{tabular}

for the synthesis of many active constituents, particularly those that are more pharmacologically active against certain diseases [19]. Gathering leaves could be promoted as a sustainable practice, since in most cases at least, a number of leaves are left on the parent plant which then allows the plants to carry on their life functions [20].

In this study, herbal remedies were found to be largely prepared by decoction and were administered mainly orally by potions $(60 \%)$ which were in agreement with the results of other studies conducted in northern Thailand $[9,18,21]$. Moreover, the results also revealed that almost all of the surveyed species are used singly as monoherbal recipes with a specific part of the plant used for a particular disease.

The ICF values varied from 0.00 to 1.00 (Table 2). The highest recorded ICF values (0.89-1.00) indicated the best level of agreement among the informants in terms of the use of the medicinal plant species reported to be used for treating carminative disorders, geographic tongue, stomachaches, constipation, as well as an appetite 
stimulant, for food poisoning (1.00 each), flatulence, as a laxative (0.97 each), for diarrhea, mouth ulcers ( 0.95 each), gastric ulcers (0.92), and jaundice (0.89). However, hemorrhoids indicated the lowest value (0.00). According to Gazzaneo et al. [16] high ICF values are important in the identification of plants of particular interest in the search for bioactive compounds.

This study reported the highest fidelity level values for Punica granatum (100.00), Psidium guajava (95.45) and Musa sapientum (81.58) against diarrhea; Dillenia pentagyna (84.62), Engelhardtia spicata var. colebrookeana (81.25) and Curcuma longa (70.63) against gastric ulcers; Zingiber montanum (90.20) and Zingiber ottensii (81.75) against gastric ulcers; Senna occidentalis (87.18), Euphorbia heterophylla (72.22) and Senna alata (70.59) as a laxative; Gymnopetalum integrifolium (90.91), Dendrocalamus strictus (75.00) against jaundice and Melastoma malabathricum (76.92) against mouth ulcers. These medicinal plants could be considered a clue for the high healing potential of these plants against the corresponding diseases. Plants with the highest fidelity level values could also be targeted for further phytochemical investigations in order to identify the bioactive components that are responsible for their high healing potential.

\section{Ethnomedicinal relevance}

Digestive system disorders are one of the most common types of ailments affecting humans. Several ethnomedicinal studies revealed that the use of medicinal plants by traditional people against digestive system disorders is a common practice throughout the world. These illnesses were the most important usage categories in many different countries and areas, such as in Ethiopia [22], Brazil [23], the Peruvian Andes [24] and Bolivia [25]. Interestingly, thirty-three (93\%) of the studied plants were found to have been used for treating digestive system disorders in other ethnomedicinal investigations in different parts of the world (Table 5). Moreover, twentythree $(64 \%)$ of the surveyed plants shared similar use aliments with other studies. The repetitive usage of these plant species used by the Karen people may serve as an indication of their effectiveness and show a high value of medicinal plant knowledge for treating digestive system disorders.

\section{Pharmacology relevance and chemical constitute}

Several investigators have reported on the pharmacological relevance of the plants used in digestive system disorders. In the present study, 19 medicinal plants were found to be pharmacologically active against digestive system disorders (Table 5). The highest numbers of pharmacological activity reported were for antiulcer activity (15 species), antidiarrhoeal activity (8 species), hepatoprotective activity ( 8 species), stimulant laxative activity (2 species) and prokinetic activity (1 species). Moreover, the ethnomedicinal uses of 12 plants were similar with the pharmacological activities reported. Therefore, these investigations might confirm that some medicinal plants have a potential effect on treating digestive system disorders.

The important medicinal plants for treating diarrhoeal diseases were Punica granatum (UV =0.71; FL=100.00), Psidium guajava $(\mathrm{UV}=0.61 ; \mathrm{FL}=95.45)$, Musa sapientum $(\mathrm{UV}=0.34 ; \mathrm{FL}=81.58)$, Leea indica $(\mathrm{UV}=0.14 ; \mathrm{FL}=$ $50.44)$, Ensete glaucum $(\mathrm{UV}=0.23 ; \mathrm{FL}=50.00)$. Several investigations revealed that tannins and other polyphenolic compounds, such as coumarins, flavonoids, triterpenoids, saponins, and a host of other plant secondary metabolites possess antidiarrhoeal properties [26-28]. Particular tannins are responsible for protein denaturation and for producing the protein tannate, which reduces secretions from intestinal mucosa [29]. Studies on the phytochemical properties showed that the crude extracts of Punica granatum seed [30] and Psidium guajava leaves [31] contain numerous tannins. Therefore, these plants may produce antisecretory activity and antidiarrhoeal activity in animals. Another pharmacological study [32] revealed that pectins, which are found in the cell wall and in intracellular substances in many fruits, had therapeutic effects on treating diarrhea. It was also reported that green bananas (Musa sapientum) were rich in pectins and could significantly reduce diarrhea in children.

The second highest use recorded among the Karen people was for flatulence. Six of eight plants used for treating this illness were from the Zingiberaceae family (Boesenbergia rotunda ( $\mathrm{UV}=0.39 ; \mathrm{FL}=65.00)$, Curcuma longa $(\mathrm{UV}=0.56 ; \quad \mathrm{FL}=5.56)$, Kaempferia parviflora $(\mathrm{UV}=0.17 ; \mathrm{FL}=31.82)$, Zingiber montanum $(\mathrm{UV}=0.72$; $\mathrm{FL}=90.20)$, Zingiber officinale $(\mathrm{UV}=0.30 ; \mathrm{FL}=18.42)$ and Zingiber ottensii $(\mathrm{UV}=0.34 ; \mathrm{FL}=81.75)$ ). A biological study revealed that the active constituents in the essential oils, such as the gingerols in zingiberaceae plants, inhibited a multiplication of the colon bacteria that ferment undigested carbohydrates causing flatulence [33].

The most prevalent plants used as laxatives were Senna occidentalis (UV = 0.59; FL = 87.18), Senna alata (UV = 0.49; FL = 70.59), Euphorbia heterophylla (UV = 0.47; FL =72.22), and Tamarindus indica ( $\mathrm{UV}=0.26$; $F L=62.75)$. The important phytochemical constituents in Senna were anthraquinones [34,35], which are known to have stimulant laxative properties [36]. Moreover, these phytochemicals are present in various drugs used in Europe. Another study on the laxative properties of medicinal plants determined the significance of Tamarindus indica [37]. It was reported that the tartaric acid, malic acid and potassium acid in this plant were major constituents inducing laxative activity. 
Table $\mathbf{5}$ Literature study of the surveyed medicinal plants and their relevant ethnomedicinal uses, pharmacological studies and known chemical constituents

\begin{tabular}{|c|c|c|c|}
\hline Scientific name & $\begin{array}{l}\text { Relevant ethnobotanical } \\
\text { citations }\end{array}$ & $\begin{array}{l}\text { Relevant pharmacological } \\
\text { citations }\end{array}$ & Known chemical constituents \\
\hline Acorus calamus L. & Stomachache* ${ }^{*}$ carminative [60] & antidiarrhoeal activity [61] & asarones [62] \\
\hline $\begin{array}{l}\text { Asparagus filicinus Buch.-Ham. } \\
\text { ex D.Don }\end{array}$ & stomachache [60], diarrhea [63] & NA & $\begin{array}{l}\text { steroidal saponins, oligospirostanosides, } \\
\text { oligofurostanosides, aspafilioside [64] }\end{array}$ \\
\hline Boesenbergia rotunda (L.) Mansf. & flutulence* ${ }^{*}$, indigestion [65] & $\begin{array}{l}\text { antiulcer activity [66], } \\
\text { hepatoprotective activity [67] }\end{array}$ & $\begin{array}{l}\text { boesenbergin, cardamonin, pinostrobin, } \\
\text { pinocembrin, alpinetin [68] }\end{array}$ \\
\hline Cassytha filiformis L. & $\begin{array}{l}\text { liver disease [69] dysentery, } \\
\text { gastric ulcer [29] }\end{array}$ & NA & $\begin{array}{l}\text { aporphine alkaloids, cassyformine, } \\
\text { filiformine [70] }\end{array}$ \\
\hline Celastrus paniculatus Willd. & laxative 71] & NA & malkanguniol [71] \\
\hline Centella asiatica (L.) Urb. & gastric ulcer* [72], diarrhea* [73] & ulcer protective effect** [74] & $\begin{array}{l}\text { centelloids, pentacyclic triterpenoid, } \\
\text { polyacetylenes, asiaticosides [75] }\end{array}$ \\
\hline Croton kongensis Gagnep. & acute gastroenteritis*[76] & NA & NA \\
\hline Croton robustus Kurz & NA & NA & $\begin{array}{l}\text { trachyloban-19-oic acid, trachyloban-19-ol, } \\
\text { poilaneic acid [77] }\end{array}$ \\
\hline Curcuma longa L. & Carminative [78] & $\begin{array}{l}\text { antiulcer activity**, } \\
\text { hepatoprotective activity [40] }\end{array}$ & $\begin{array}{l}\text { curcuminoid [79], zingiberene, } \\
\text { sesquiterpenes, a-phellandrene [78] }\end{array}$ \\
\hline Dendrocalamus strictus (Roxb.) Nees & dysentery [80] & NA & NA \\
\hline Dillenia pentagyna Roxb. & diarrhea [81], jaundice [82] & NA & $\begin{array}{l}\text { betulinic acid, naringenin 7-galactosyl, } \\
\text { dihedral quercetin 5-glactoside [83] }\end{array}$ \\
\hline Embelia sessiliflora Kurz & diarrhea [62] & NA & NA \\
\hline $\begin{array}{l}\text { Engelhardtia spicata Blume var. } \\
\text { colebrookeana (Lindl. ex Wall.) } \\
\text { Kuntze }\end{array}$ & mouth ulcer [21], diarrhea [62] & NA & Engelhardtione, oleanolic acid [84] \\
\hline Ensete glaucum (Roxb.) Cheesman & $\begin{array}{l}\text { gastritis, constipation [9], food } \\
\text { poisoning* }[60]\end{array}$ & NA & NA \\
\hline Euphorbia heterophylla L. & laxative*, constipation [85] & NA & $\begin{array}{l}\text { stigmasterol, stigmasterol glucoside, } \\
\text { benzoic acid, } 4 \text { - hydroxyl benzoic } \\
\text { acid [85] }\end{array}$ \\
\hline Euphorbia hirta L. & vomitting [86], diarrhea [73] & antidiarrhoeal activity [87] & quercitrin, myricitrin, Afzelin [88] \\
\hline $\begin{array}{l}\text { Flacourtia jangomas (Lour.) } \\
\text { Raeusch. }\end{array}$ & $\begin{array}{l}\text { toothache* [89], diarrhea* [90] } \\
\text { liver disease [91] }\end{array}$ & antidiarrhoeal activity** [92] & phenolics, tannins, terpenoids [93] \\
\hline $\begin{array}{l}\text { Flemingia macrophylla (Willd.) } \\
\text { Merr. }\end{array}$ & indigestive, flatulence [90] & hepatoprotective activity ${ }^{* *}$ [39] & fleminone, flemiphyllin [94] \\
\hline Gmelina arborea Roxb. & $\begin{array}{l}\text { diarrhea [95] stomach-hepatic } \\
\text { diseases* }[96]\end{array}$ & $\begin{array}{l}\text { antidiarrhoeal activity [97], } \\
\text { hepatoprotective activity [98] }\end{array}$ & $\begin{array}{l}\text { monoacylated, diacylated, triacylated } \\
\text { iridoid glycosides [90] }\end{array}$ \\
\hline Gymnopetalum integrifolium Kurz. & NA & NA & cucurbitacin [99] \\
\hline Kaempferia parviflora Wall. ex Baker & gastric ulcer* [100] & antiulcer activity** $^{* *}$ [42] & $\begin{array}{l}\text { methoxyflavones (5,7,4' trimethoxyflavone, } \\
\text { 5,7-dimethoxyflavone) [100] }\end{array}$ \\
\hline Leea indica (Burm. f.) Merr. & diarrhea* [69] & NA & $\begin{array}{l}\text { flavonids, leucoanthocyanidins, galic acid, } \\
\text { amorphous froth forming acid [101] }\end{array}$ \\
\hline Melastoma malabathricum L. & $\begin{array}{l}\text { diarrhea, dysentery [102], } \\
\text { toothache [103] }\end{array}$ & antidiarrhoeal activity [102] & $\begin{array}{l}\text { ellagic acid, anthocyanin, nobotannin } \\
\text { B [103] }\end{array}$ \\
\hline Musa sapientum L. & $\begin{array}{l}\text { gastric ulcer [104], diarrhea*, } \\
\text { dysentery [105] }\end{array}$ & $\begin{array}{l}\text { antidiarrhoeal activity* [32], } \\
\text { antiulcer activity [106] }\end{array}$ & $\begin{array}{l}\text { alkaloids, pectins, flavonoids, } \\
\text { catecholamines, acyl steryl glycosides } \\
\text { [105] }\end{array}$ \\
\hline Mussaenda sanderiana Ridl. & laxative, toothache ${ }^{*}[90]$ & NA & NA \\
\hline Ochna integerrima (Lour.) Merr. & $\begin{array}{l}\text { jaundice*, mouth ulcer [21] } \\
\text { digestive tonic [107] }\end{array}$ & NA & $\begin{array}{l}\text { ochnaflavone, dihydroochnaflavone, } \\
\text { lophirone, calodenone [107] }\end{array}$ \\
\hline Psidium guajava L. & diarrhea* [108] & antidiarrhoeal activity $^{* *}$ [57] & $\begin{array}{l}\text { tannins, polyphenolic compounds, } \\
\text { triterpenoid, guajanoic acid [31] }\end{array}$ \\
\hline Punica granatum L. & diarrhea* [109] & antidiarrhoeal activity ${ }^{* *}$ [26] & $\begin{array}{l}\text { punicalagins, ellagic acid, tannin, } \\
\text { anthocyanins [30] }\end{array}$ \\
\hline
\end{tabular}




\begin{tabular}{|c|c|c|c|}
\hline Senna alata (L.) Roxb. & laxative* ${ }^{*}$ colon cleanser [110] & $\begin{array}{l}\text { stimulant laxative activities** } \\
\text { and againt costipation [36] }\end{array}$ & $\begin{array}{l}\text { anthraquinoes, phenols, tannins, } \\
\text { saponins, flavonoids [34] }\end{array}$ \\
\hline Senna occidentalis (L.) Link & $\begin{array}{l}\text { laxative* [111], liver disease [112] } \\
\text { gastrointestinal disease [113] }\end{array}$ & stimulant laxative activities ${ }^{* *}[50]$ & anthaquinones [35] \\
\hline Tamarindus indica $\mathrm{L}$. & laxative* [114], gastric ulcer [112] & $\begin{array}{l}\text { stimulant laxative activities** } \\
\text { [37] antiulcer activity [115] }\end{array}$ & $\begin{array}{l}\text { tamarindienal, tartaric acid, malic } \\
\text { and tartaric acids potassium acid } \\
\text { [37] }\end{array}$ \\
\hline Thunbergia laurifolia Lindl. & stomachache, carminative [9] & hepatoprotective effects** [116] & $\begin{array}{l}\text { iridoid glucosides, delphinidin, } \\
\text { apigenin [52] }\end{array}$ \\
\hline $\begin{array}{l}\text { Zingiber montanum (J.König) } \\
\text { Link ex A.Dietr. }\end{array}$ & flatulence* ${ }^{*}$ carminative [5] & antiulcer activity* [41] & $\begin{array}{l}\text { terpinen-4-ol, phenylbutenoids, } \\
\text { zerumbone [52] }\end{array}$ \\
\hline Zingiber officinale Roscoe & flatulence $^{*}[113]$ & $\begin{array}{l}\text { prokinetic activity [117] } \\
\text { hepatoprotective activity [118] }\end{array}$ & gingerols, shogaols [119] \\
\hline Zingiber ottensii Valeton & jaundice $[120]$ & NA & $\begin{array}{l}\text { zerumbone, terpinen-4-ol, p-cymene } \\
\text { [52] }\end{array}$ \\
\hline Ziziphus cambodiana Pierre & NA & NA & $\begin{array}{l}\text { triterpenes, saponins, cyclopeptide, } \\
\text { alkaloids [121] }\end{array}$ \\
\hline
\end{tabular}

*ethnomedicinal knowledge in other ethnic groups which have similar usage to medicinal plants used by the Karen. ** pharmacological study which is relevant to the medicinal plants used by the Karen. NA: data not available.

Jaundice is an ailment characterized by a yellowish pigmentation of the skin, the conjunctival membranes over the sclerae (the whites of the eyes), and other mucous membranes [38]. It is often seen in liver diseases such as hepatitis or liver cancer. The use of medicinal plants to treat jaundice was also recorded as high in this study. The important plants used for treating this ailment were Dendrocalamus strictus ( $\mathrm{UV}=0.36 ; \mathrm{FL}=75.00)$, Flemingia macrophylla (UV=0.28; $\mathrm{FL}=46.15)$, Croton robustus $(\mathrm{UV}=0.21 ; \mathrm{FL}=56.25)$, Gymnopetalum integrifolium ( $\mathrm{UV}=0.19 ; \mathrm{FL}=90.91$ ), and Mussaenda sanderiana $(\mathrm{UV}=0.17 ; \mathrm{FL}=44.19)$. Interestingly, most medicinal plants used for treating jaundice were administrated by bath and potion. This reflects the culturally herbal administration for treating specific aliments by the Karen people. Moreover, a pharmacological study revealed that the aqueous extract of Flemingia macrophylla had a hepatoprotective effect against liver damage in rats [39].

A high use for the treatment of gastric ulcers was also recorded (168 uses) among the Karen people. Several plants were used for treating this disease, including Engelhardtia spicata var. colebrookeana (UV $=0.59$; $\mathrm{FL}=81.25)$, Curcuma longa $(\mathrm{UV}=0.56 ; \mathrm{FL}=70.63)$, Dillenia pentagyna $(\mathrm{UV}=0.52 ; \mathrm{FL}=84.62)$, Croton kongensis ( $\mathrm{UV}=0.30 ; \mathrm{FL}=42.31$ ), Ziziphus cambodiana $(\mathrm{UV}=0.16 ; \mathrm{FL}=36.36)$, Kaempferia parviflora $(\mathrm{UV}=0.17 ; \mathrm{FL}=13.64)$, Zingiber montanum $(\mathrm{UV}=0.72$; $\mathrm{FL}=13.64$ ). A pharmacological study on Curcuma longa [40] found that curcumin, which was the active constituent in this plant, had a beneficial effect on the stomach. It could block indomethacin, ethanol in stress-induced gastric ulcers and could also prevent pylorus-ligation-induced acid secretion in rats. Moreover, the antiulcer activity study on Zingiber montanum reported that zerumbone, which was the important phytochemical property of this plant, showed potent cytoprotective and antiulcerogenic effects against hydrochloric acid $(\mathrm{HCl})$ induced gastric ulceration in mice [41]. Another pharmacological investigation involved Kaempferia parviflora [42]. Its ethanolic extract could increase gastric mucus secretions, which were related to the preservation of ulcer-damaged tissue.

Overall, several plants displayed effective biological activities against different digestive aliments. However, a literature search found that some medicinal plants used by the Karen people have not been included in any pharmacological studies on the digestive system. Therefore, it is of significant interest to investigate the biological research of some of the Karen people's medicinal plants used for the treatment of digestive system disorders.

\section{Toxicity of medicinal plants}

A literature investigation found that 18 of the 36 species named had been previously exposed to a toxicology study (Table 4). Six species (Centella asiatica, Curcuma longa, Gmelina arborea, Psidium guajava, Punica granatum and Tamarindus indica) had no toxic effect on animals, whereas 12 species showed a toxic effect. The different toxic effects recorded after administrating medicinal plants were found in the digestive system (Flemingia macrophylla, Kaempferia parviflora and Senna occidentals), the blood cells and the circulatory system (Euphorbia heterophylla, Euphorbia hirta, Senna alata and Thunbergia laurifolia), the nervous system (Celastrus paniculatus), and the reproductive system (Zingiber 
officinale). Moreover, a study on the acute toxicity of these treatments on mice found that Zingiber montanum had the highest toxicity level $\left(\mathrm{LD}_{50}=80 \mathrm{~g} / \mathrm{kg}\right)$.

Most Karen people believe that medicinal plants do not produce any side effects. They are also cheap and locally available. However, natural products may also contain a few harmful ingredients as secondary metabolites [43] which may produce perilous side effects. Therefore, medicinal plants must be taken in the proper amounts and long-term administration must be avoided for the optimal health and well-being of the patient.

\section{Conclusion}

Digestive system disorders have a high prevalence in terms of the morbidity rate among Thai people. This is also considered to be true worldwide, particularly among ethnic people who likely have inadequate access to hygienic levels of sanitation, which may increase the transmission of digestive diseases. The study of medicinal plants among the Karen people of northern Thailand has reported that 36 species were commonly used against digestive system disorders. A literature investigation found that several surveyed plants had similar usage with other ethnic groups in different areas throughout the world. Moreover, the pharmacological studies of some of the medicinal plants could confirm that these plants are considered effective in treating digestive diseases. However, some medicinal plants, which were reported to have high UV and FL values, still require further pharmacological research for the discovery of new compounds and biological activities of these potential medicinal plants. There were certain toxic effects that were found to have been associated with some of these plants. Therefore, herbal remedies should be taken carefully in order to avoid any potential side effects that may occur through utilizing these medicinal plants.

\section{Competing interests}

The authors declare that they have no competing interests.

\section{Authors' contributions}

KT, KK and TS carried out field research. CT and CW supervised the work. KT analyzed the data and wrote the manuscript. All authors approved the final version of this manuscript.

\section{Acknowledgments}

We thank the Karen people of northern Thailand for sharing their knowledge of medicinal plants with us and for permitting us to publish our findings. We thank the Royal Project Foundation for transportation and accommodations during our field work. We would also like to thank the Office of the Higher Education Commission of Thailand for funding of this PhD study.

\section{Author details}

'Department of Biology, Faculty of Science, Chiang Mai University, Huaykaew Road, Chiang Mai 50200, Thailand. ²Royal Park Rajapruek, Mae-hea, Muang, Chiang Mai 50200, Thailand.

Received: 26 June 2014 Accepted: 9 February 2015

Published online: 09 April 2015

\section{References}

1. WHO. World health statistics 2014. Switzerland: WHO Press; 2014.

2. Balick MJ. Transforming ethnobotany for the new millennium. Ann Missouri Bot Gard. 1996;83:58-66.

3. Akerele O. WHO's traditional medicine programme, progress and perspectives. WHO Chron. 1984;38:76-81.

4. Balick MJ, Cox PA. Ethnobotanical research and traditional healthcare in developing countries. In: Bodeker G, Bhat KKS, Burley J, Vantomme P, editors. Medicinal Plant for Forest Conservation and HealthCare. Rome: Food and Agriculture Organization of the United Nation; 1997.

5. Anderson EF. Plants and people of the golden triangle. Hong Kong: Dioscorides Press; 1993.

6. Ministry of Public Health. Public health statistics 2010. Bangkok: The War Veterans Organization of Thailand Press; 2014.

7. Junsongduang A, Balslev H, Inta A, Jampeetong A, Wangpakapattanawong P. Karen and lawa medicinal plant use: uniformity or ethnic divergence? J Ethnopharm. 2014;151:517-27.

8. Inta A, Shengji P, Balslev H, Wangpakapattanawong P, Trisonthi C. A comparative study on medicinal plants used in Akha's traditional medicine in China and Thailand, cultural coherence or ecological divergence? J Ethnopharm. 2008;116:508-17.

9. Inta A, Trisonthi P, Trisonthi C. Analysis of traditional knowledge in medicinal plants used by Yuan in Thailand. J Ethnopharm. 2013;149:344-51.

10. Trisonthi $C$, Trisonthi P. Ethnobotanical study in Thailand, A case study in Khun Yuam District Maehongson Province. Thai J Bot. 2009;1:1-23.

11. Department of Social Development and Welfare. The population of communities in highland in 20 provinces, Thailand. Bangkok: Ministry of Social Development and Human Security; 2002.

12. Perve E. The Hill Tribes Living in Thailand. Chiang Mai, Thailand: Prachakorn; 2006.

13. Asavachaichan S. Chiang Mai. Bangkok: Sarakadee Press; 2010.

14. Phillips O, Gentry AH, Reynel C, Wilkin P, Galvez-Durand BC. Quantitative ethnobotany and Amazonian conservation. Conserv Biol. 1994:8:225-48.

15. Trotter RT, Logan MH. Informant consensus: a new approach for identifying potentially effective medicinal plants. In: Etkin NL, editor. Plants in Indigenous Medicine and Diet. Bedford Hills, New York: Redgrave Publishing Company; 1986. p. 91-112.

16. Gazzaneo L, de Lucena R, de Albuquerque U. Knowledge and use of medicinal plants by local specialists in an region of Atlantic Forest in the state of Pernambuco (Northeastern Brazil). J Ethnobiol Ethnomed. 2005;1:9.

17. Friedman J, Yaniv Z, Dafni A, Palewitch D. A preliminary classification of the healing potential of medicinal plants, based on a rational analysis of an ethnopharmacological field survey among Bedouins in the Negev Desert, Israel. J Ethnopharm. 1986;16:275-87.

18. Srithi K, Balslev H, Wangpakapattanawong P, Srisanga P, Trisonthi C. Medicinal plant knowledge and its erosion among the Mien (Yao) in northern Thailand. J Ethnopharm. 2009;123:335-42.

19. Passalacqua NG, Guarrera PM, De Fine G. Contribution to the knowledge of the folk plant medicine in Calabria region (Southern Italy). Fitoterapia. 2007;78:52-68.

20. Lulekal E, Asfaw Z, Kelbessa E, Van Damme P. Ethnomedicinal study of plants used for human ailments in Ankober District, North Shewa Zone, Amhara Region. Ethiopia J Ethnobiol Ethnomed. 2013;9:63.

21. Khuankaew S, Srithi K, Tiansawat $P$, Jampeetong A, Inta A, Wangpakapattanawong P. Ethnobotanical study of medicinal plants used by Tai Yai in Northern Thailand. J Ethnopharm. 2014;151:829-38.

22. Giday M, Asfaw Z, Woldu Z. Medicinal plants of the meinit ethnic group of Ethiopia: an ethnobotanical study. J Ethnopharm. 2009;124:513-21.

23. De Albuquerque UP, De Medeiros PM, De Almeida ALS, Monteiro JM, Neto EMDFL, De Melo JG, et al. Medicinal plants of the caatinga (semi-arid) vegetation of NE Brazil: a quantitative approach. J Ethnopharm. 2007;114:325-54.

24. De-La-Cruz H, Vilcapoma G, Zevallos PA. Ethnobotanical study of medicinal plants used by the Andean people of Canta, Lima, Peru. J Ethnopharm. 2007;111:284-94.

25. Bourdy G, DeWalt SJ, de Michel LR C, Roca A, Deharo E, Muñoz V, et al. Medicinal plants uses of the Tacana, an Amazonian Bolivian ethnic group. J Ethnopharm. 2000;70:87-109.

26. Das AK, Mandal SC, Banerjee SK, Sinha S, Das J, Saha BP, et al. Studies on antidiarrhoeal activity of Punica granatum seed extract in rats. J Ethnopharm. 1999;68:205-8. 
27. Mbagwu HOC, Adeyemi OO. Anti-diarrhoeal activity of the aqueous extract of Mezoneuron benthamianum Baill (Caesalpiniaceae). J Ethnopharm y. 2008;116:16-20.

28. Suleiman MM, Dzenda T, Sani CA. Antidiarrhoeal activity of the methanol stem-bark extract of Annona senegalensis Pers. (Annonaceae). J Ethnopharm. 2008;116:125-30

29. Tripathi YC, Prabhu W, Pal RS, Mishra RN. Medicinal plants of Rajasthan in Indian system of medicine. Anc sci life. 1996;15:190-212.

30. Jurenka JS. Therapeutic applications of pomegranate (Punica granatum L.): a review. Altern Med Rev. 2008;13:128-44.

31. Begum S, Hassan SI, Ali SN, Siddiqui BS. Chemical constituents from the leaves of Psidium guajava. Nat Prod Res. 2004;18:135-40.

32. Rabbani GH, Teka T, Zaman B, Majid N, Khatun M, Fuchs GJ. Clinical studies in persistent diarrhea: dietary management with green banana or pectin in Bangladeshi children. Gastroenterology. 2001;121:554-60.

33. Nigam N, George J, Shukla Y. Ginger (6-gingerol). In: Aggarwal BB, Kunnumakkara $A B$, editors. Molecular targets and therapeutic uses of spices, modern uses for ancient medicine. Singapore: World Scientific Publishing Co. Pte. Ltd; 2009. p. 225-56.

34. Sule WF, Okonko IO, Omo-Ogun S, Nwanze JC, Ojezele MO, Ojezele OJ, et al. Phytochemical properties and in-vitro antifungal activity of Senna alata Linn. crude stem bark extract. J Med Plants Res. 2011;5:176-83.

35. Yadav JP, Arya V, Yadav S, Panghal M, Kumar S, Dhankhar S. Cassia occidentalis L. A review on its ethnobotany, phytochemical and pharmacological profile. Fitoterapia. 2010;81:223-30.

36. Hennebelle T, Weniger B, Joseph H, Sahpaz S, Bailleul F. Senna alata. Fitoterapia. 2009;80:385-93.

37. Bhadoriya S, Ganeshpurkar A, Narwaria J, Rai G, Jain AP. Tamarindus indica: extent of explored potential. Pharmacogn Rev. 2011;5:73-81.

38. Click R, Dahl-Smith J, Fowler L, DuBose J, Deneau-Saxton M, Herbert J. An osteopathic approach to reduction of readmissions for neonatal jaundice. Osteopath Fam Physician. 2013;5:17-23.

39. Hsieh P-C, Ho Y-L, Huang G-J, Huang M-H, Chiang Y-C, Huang S-S, et al. Hepatoprotective effect of the aqueous extract of Flemingia macrophylla on carbon tetrachloride-induced acute hepatotoxicity in rats through anti-oxidative activities. Am J Chin Med. 2011;39:349-65.

40. Chattopadhyay I, Biswas K, Bandyopadhyay U, Banerjee RK. Turmeric and curcumin: biological actions and medicinal applications. Curr Sci. 2004:87:1325-5.

41. Al-Amin M, Sultana GNN, Hossain CF. Antiulcer principle from Zingiber montanum. J Ethnopharm. 2012;141:57-60.

42. Rujjanawate C, Kanjanapothi D, Amornlerdpison D, Pojanagaroon S. Anti-gastric ulcer effect of Kaempferia paniflora. J Ethnopharm. 2005;102:120-2.

43. Singh $P$, Singh A. Acute toxic effects of Medicinal Plant Jatropha gossypifolia on non- target fish and mice. J Agr Res. 2012;1:433-8.

44. Dandiya PC, Cullumbine H. Studies on Acorus calamus (III): some pharmacological actions of the volatile oil. J Pharmacol Exp Ther. 1959;125:353-9.

45. Libman A, Bouamanivong S, Southavong B, Sydara K, Soejarto DD. Medicinal plants: an important asset to health care in a region of central Laos. J Ethnopharm. 2006;106:303-11.

46. Nalini K, Karanth KS, Rao A, Aroor AR. Effects of Celastrus paniculatus on passive avoidance performance and biogenic amine turnover in albino rats. J Ethnopharm. 1995;47:101-8

47. Adedapo AA, Abatan MO, Olorunsogo OO. Toxic effects of some plants in the genus Euphorbia on haematological and biochemical parameters of rats. Vet Arhiv. 2004;74:53-62

48. Syiem D, Khup PZ. Evaluation of Flemingia macrophylla L., A traditionally used plant of the north eastern region of India for hypoglycemic and anti-hyperglycemic effect on mice. Pharmacologyonline. 2007;2:355-66.

49. Sudwan P, Saenphet K, Saenphet S, Suwansirikul S. Effect of Kaempferia parviflora Wall. ex. Baker on sexual activity of male rats and its toxicity. Southeast Asian J Trop Med Public Health. 2006;37:210-5.

50. Nadal SR, Calore EE, Manzione CR, Puga FR, Perez NM. Effects of long-term administration of Senna occidentalis seeds in the large bowel of rats. Pathol Res Pract. 2003;199:733-7.

51. Chivapat S, Chavalittumrong P, Attawish A, Bansiddhi J, Padungpat S. Chronic toxicity of Thunbergia laurifolia Lindl. extract. J Thai Trad Altern Med. 2009;7:17-24.
52. Areekul S, Intorn J, Takheaw S, Nantakaw A. Wild plant knowledge used in northern Thailand III. Bangkok: Amarin Printing \& Publishing Public Company Limited; 2009.

53. Weidner MS, Sigwart K. Investigation of the teratogenic potential of a Zingiber officinale extract in the rat. Reprod Toxicol. 2001;15:75-80.

54. Brinkhaus B, Lindner M, Schuppan D, Hahn EG. Chemical, pharmacological and clinical profile of the East Asian medical plant Centella asiatica. Phytomedicine. 2000;7:427-48.

55. Chainani-Wu N. Safety and anti-inflammatory activity of curcumin: a component of tumeric (Curcuma longa). J Altern Complem Med. 2003;9:161-8.

56. Kulkarni Y, Veeranjaneyulu A. Toxicological studies on aqueous extract of Gmelina arborea in rodents. Pharm Biol. 2010;48:1413-20.

57. Ojewole JA, Awe EO, Chiwororo WD. Antidiarrhoeal activity of Psidium guajava Linn. (Myrtaceae) leaf aqueous extract in rodents. J Smooth Muscle Res. 2008;44:195-207.

58. Cerda B, Ceron JJ, Tomas-Barberan FA, Espin JC. Repeated oral administration of high doses of the pomegranate ellagitannin punicalagin to rats for 37 days is not toxic. J Agr Food Chem. 2003;51:3493-501.

59. Komutarin T, Azadi S, Butterworth L, Keil D, Chitsomboon B, Suttajit M, et al. Extract of the seed coat of Tamarindus indica inhibits nitric oxide production by murine macrophages in vitro and in vivo. Food Chem Toxicol. 2004;42:649-58.

60. Ghorbani A, Langenberger G, Feng L, Sauerborn J. Ethnobotanical study of medicinal plants utilised by Hani ethnicity in Naban River Watershed National Nature Reserve, Yunnan, China. J Ethnopharm. 2011;134:651-67.

61. Shoba FG, Thomas M. Study of antidiarrhoeal activity of four medicinal plants in castor-oil induced diarrhoea. J Ethnopharm. 2001;76:73-6.

62. Areekul S, Intorn J, Takheaw S, Nantakaw A. Wild plant knowledge used in northern Thailand I. Bagnkok: Amarin Printing \& Publishing Public Company Limited; 2009.

63. Bhat JA, Kumar M, Bussmann RW. Ecological status and traditional knowledge of medicinal plants in Kedarnath Wildlife Sanctuary of Garhwal Himalaya, India. J Ethnobiol Ethnomed. 2013;9:1.

64. Sharma SC, Thakur NK. Oligofurostanosides and oligospirostanosides from roots of Asparagus filicinus. Phytochem. 1996;41:599-603.

65. Wan-Ibrahim WI, Sidik K, Kuppusamy UR. A high antioxidant level in edible plants is associated with genotoxic properties. Food Chem. 2010;122:1139-44.

66. Abdelwahab SI, Mohan S, Abdulla MA, Sukari MA, Abdul AB, Taha MME, et al. The methanolic extract of Boesenbergia rotunda (L.) Mansf. and its major compound pinostrobin induces anti-ulcerogenic property in vivo: Possible involvement of indirect antioxidant action. J Ethnopharm. 2011;137:963-70.

67. Salama SM, Abdulla MA, AIRashdi AS, Hadi AHA. Mechanism of hepatoprotective effect of Boesenbergia rotunda in thioacetamide-induced liver damage in rats. Evid-Based Compl Alt. 2013;2013:157456.

68. Ching AYL, Wah TS, Sukari MA, Lian GEC, Rahmani M, Khalid K. Characterization of flavonoid derivatives from Boesenbergia rotunda (L.). Malays J Anal Sci. 2007;11:154-9.

69. Jain A, Katewa SS, Galav PK, Sharma P. Medicinal plant diversity of Sitamata wildlife sanctuary, Rajasthan, India. J Ethnopharm. 2005;102:143-57.

70. Chang F-R, Chao Y-C, Teng C-M, Wu Y-C. Chemical Constituents from Cassytha filiformis II. J Nat Prod. 1998;61:863-6.

71. Bhanumathy M, Chandrasekar SB, Chandur U, Somasundaram T. Phyto-pharmacology of Celastrus paniculatus: an Overview. Int J Pharm Sci Rev Res. 2010;2:176-81.

72. Noumi E, Dibakto TW. Medicinal plants used for peptic ulcer in the Bangangte region, western Cameroon. Fitoterapia. 2000;71:406-12.

73. Kala CP. Ethnomedicinal botany of the Apatani in the Eastern Himalayan region of India. J Ethnobiol Ethnomed. 2005;1:11.

74. Sairam K, Rao CV, Goel RK. Effect of Centella asiatica Linn on physical and chemical factors induced gastric ulceration and secretion in rats. Indian J Exp Biol. 2001;39:137-42.

75. Siddiqui BS, Aslam H, Ali ST, Khan S, Begum S. Chemical constituents of Centella asiatica. J Asian Nat Prod Res. 2007;9:407-14.

76. Lai $X$, Yang $Y$, Shan $X$. The investigation of Euphorbiaceous medicinal plants in Southern China. Econ Bot. 2004;58:S307-20.

77. Ngamrojnavanich N, Tonsiengsom S, Lertpratchya P, Roengsumran S, Puthong S, Petsom A. Diterpenoids from the Stem Barks of Croton robustus. Arch Pharm Re. 2003;26:898-901. 
78. Eigner D, Scholz D. Ferula asa-foetida and Curcuma longa in traditional medical treatment and diet in Nepal. J Ethnopharm. 1999;67:1-6.

79. Roth GN, Chandra A, Nair MG. Novel Bioactivities of Curcuma longa constituents. J Nat Prod. 1998:61:542-5.

80. Kiruba S, Jeeva S, Dhas SSM. Enumeration of ethnovetenerinary plant of Cape Comorin, Tamil Nadu. Indian J Tradit Knowl. 2006:5:576-8.

81. Dubey PC, Sikarwar RLS, Khanna KK, Tiwari AP. Ethnobotany of Dillenia pentagyna Roxb. In Vindhya region of Madhya Pradesh, India. Nat Prod Radiance. 2009;8:546-8.

82. Patil MV, Patil DA. Some herbal remedies used by the tribals of Nasik district, Maharashtra. Nat Prod Radiance. 2007;6:152-7.

83. Haque E, Islam N, Hossain M, Mohamad AU, Karim F, Rahman A. Antimicrobial and cytotoxic activities of Dillenia pentagyna. Dhaka Univ J Pharmarm Sci. 2008;7:103-5.

84. Kunwar RM, Shrestha KP, Bussmann RW. Traditional herbal medicine in Far-west Nepal: a pharmacological appraisal. J Ethnobiol Ethnomed. 2010;6:35.

85. Falodun A, Ali S, Quadir IM, Choudhary IMI. Phytochemical and biological investigation of chloroform and ethylacetate fractions of Euphorbia heterophylla leaf ( Euphorbiaceae). J Med Plants Res. 2008;2:365-9.

86. Joshi AR, Joshi K. Indigenous knowledge and uses of medicinal plants by local communities of the Kali Gandaki Watershed Area, Nepal. J Ethnopharmacol. 2000;73:175-83.

87. Tona L, Kambu K, Mesia K, Cimanga K, Apers S, De Bruyne T, et al. Biological screening of traditional preparations from some medicinal plants used as antidiarrhoeal in Kinshasa, Congo. Phytomedicine. 1999;6:59-66.

88. Kumar S, Malhotra R, Kumar D. Euphorbia hirta: Its chemistry, traditional and medicinal uses, and pharmacological activities. Pharmacogn Rev. 2010;4:58-61.

89. Khurnbongmayum AO, Khan ML, Tripathi RS. Ethnomedicinal plants in the sacred groves of Manipur. Indian J Tradit Knowl. 2005:4:21-32.

90. Areekul S, Intorn J, Takheaw S, Nantakaw A. Wild plant knowledge used in northern Thailand II. Bangkok: Amarin Printing \& Publishing Public Company Limited; 2009.

91. Govind P. Medicinal plants against liver disease. Int Res J Pharmacy. 2011:2:115-21.

92. Talukder C, Saha S, Adhikari S, Mondal HK, Islam MK, Anisuzzman M. Evaluation of antioxidant, anal gesic and antidiarrhoeal activity of Flacourtia jangomas (Lour.) Raeusch. Leaves. Pharmacologyonline. 2002;3:20-8.

93. Sreejith M, Kannappan N, Santhiagu A, Mathew AP. Phytochemical, Antioxidant and Anthelmintic activities of various leaf extracts of Flacourtia sepiaria Roxb. Asian Pac J Trop Biomed. 2013;3:947-53.

94. Ghalot K, Lal VK, Jha S. Phytochemical and pharmacological potential of Flemingia Roxb. ex W.T.Aiton (Fabaceae). Int J Phytomed. 2011;3:294-307.

95. Lawal IO, Uzokwe NE, Igboanugo ABI, Adio AF, Awosan EA, Nwogwugwu $\mathrm{JO}$, et al. Ethno medicinal information on collation and identification of some medicinal plants in Research Institutes of South-west Nigeria. Afr J Pharm Pharmaco. 2010:4:1-7.

96. Kala CP, Farooquee NA, Dhar U. Prioritization of medicinal plants on the basis of available knowledge, existing practices and use value status in Uttaranchal. India Biodivers Conserv. 2004;13:453-69.

97. Agunu A, Yusuf A, Andrew GO, Zezi AU, Abdurahman EM. Evaluation of five medicinal plants used in diarrhoea treatment in Nigeria. J Ethnopharm. 2005;101:27-30.

98. Sinha S, Dixit P, Bhargava S, Devasagayam TPA, Ghaskadbi S. Bark and fruit extracts of Gmelina arborea protect liver cells from oxidative stress. Pharm Biol. 2006;44:237-43.

99. Sekine T, Kurihara H, Waku M, Ikegami F, Ruangrungsi N. A new pentacyclic cucurbitane glucoside and a new triterpene from the fruits of Gymnopetalum integrifolium. Chem Pharm Bull. 2002;50:645-8.

100. Yenjai C, Prasanphen K, Daodee S, Wongpanich V, Kittakoop P. Bioactive flavonoids from Kaempferia parviflora. Fitoterapia. 2004;75:89-92.

101. Srinivasan GV, Ranjith C, Vijayan KK. Identification of chemical compounds from the leaves of Leea indica. Acta Pharmaceut. 2008;58:207-14.

102. Sunilson JAJ, Anandarajagopal K, Kumari AVAG, Mohan S. Antidiarrhoeal Activity of Leaves of Melastoma malabathricum Linn. Indian J Pharm Sci. 2009;71:691-7.

103. Joffry SM, Yob NJ, Rofiee MS, Affandi MM, Suhaili Z, Othman F, et al. Melastoma malabathricum (L.) Smith ethnomedicinal Uses, chemical constituents, and pharmacological properties: A Review. Evid Based Complement Alternat Med. 2012;2012:258434.
104. Dharmani PPG. Exploring Indian medicinal plants for antiulcer activity. Indian J Pharmacol. 2005;38:95-9.

105. Imam MZ, Akter S. Musa paradisiaca L. and Musa sapientum L.: A phytochemical and pharmacological review. J Appl Pharm Sci. 2001;1:14-20.

106. Goel RK, Sairam K. Anti-ulcer drugs from indigenous sources with emphasis on Musa sapientum, Tamrabhasma, Asparagus racemosus and Zingiber officinale. Indian J Pharmacol. 2002;34:100-10.

107. Bandi AKR, Lee DU, Tih RG, Gunasekar D, Bodo B. Phytochemical and biological studies of Ochna species. Chem Biodivers. 2012;9:251-71.

108. Ssegawa P, Kasenene JM. Medicinal plant diversity and uses in the Sango bay area, Southern Uganda. J Ethnopharm. 2007;113:521-40.

109. Rokaya MB, Munzbergova Z, Timsina B. Ethnobotanical study of medicinal plants from the Humla district of western Nepal. J Ethnopharm. 2010;130:485-504.

110. Clement YN, Morton-Gittens J, Basdeo L, Blades A, Francis MJ, Gomes N, et al. Perceived efficacy of herbal remedies by users accessing primary healthcare in Trinidad. BMC Complement Altern Med. 2007;7:4.

111. Jiofack T, Fokunang C, Guedje N, Kemeuze V, Fongnzossie E, Nkongmeneck $B A$, et al. Ethnobotanical uses of medicinal plants of two ethnoecological regions of Cameroon. Int J Med Med Sci. 2010;2:60-79.

112. Cano $\mathrm{JH}$, Volpato $\mathrm{G}$. Herbal mixtures in the traditional medicine of Eastern Cuba. J Ethnopharm. 2004;90:293-316.

113. Pandikumar $P$, Chellappandian M, Mutheeswaran S, Ignacimuthu S. Consensus of local knowledge on medicinal plants among traditional healers in Mayiladumparai block of Theni District, Tamil Nadu, India. J Ethnopharm. 2011;134:354-62.

114. El-Kamali HH, El-Khalifa KF. Folk medicinal plants of riverside forests of the Southern Blue Nile district, Sudan. Fitoterapia. 1999;70:493-7.

115. Raja NRL, Jegan N, Wesley J. Antiulcerogenic activity of alcoholic extract of the leaves of Tamarindus indica $(\mathrm{L})$ on experimental ulcer models. Pharmacologyonline. 2008;3:85-92.

116. Wonkchalee $\mathrm{O}$, Boonmars T, Aromdee C, Laummaunwai P, Khunkitti W, Vaeteewoottacharn K, et al. Anti-inflammatory, antioxidant and hepatoprotective effects of Thunbergia laurifolia Linn. on experimental opisthorchiasis. Parasitol Res. 2012;111:353-9.

117. Ghayur MN, Gilani AH. Pharmacological basis for the medicinal use of ginger in gastrointestinal disorders. Dig Dis Sci. 2005;50:1889-97.

118. Ajith TA, Hema U, Aswathy MS. Zingiber officinale Roscoe prevents acetaminophen-induced acute hepatotoxicity by enhancing hepatic antioxidant status. Food Chem Toxicol. 2007;45:2267-72.

119. Ali BH, Blunden G, Tanira MO, Nemmar A. Some phytochemical, pharmacological and toxicological properties of ginger (Zingiber officinale Roscoe): A review of recent research. Food Chem Toxicol. 2008;46:409-20.

120. Chuakul W, Boonpleng A. Ethnomedical uses of Thai Zingiberaceous plant (1). Thai J Phytopharmacy. 2003;10:33-9.

121. Suksamrarn $S$, Panseeta $P$, Kunchanawata $S$, Distaporn T, Ruktasing $S$, Suksamrarn A. Ceanothane- and lupane-type triterpenes with antiplasmodial and antimycobacterial activities from Ziziphus cambodiana. Chem Pharm Bull. 2006;54:535-7.

\section{Submit your next manuscript to BioMed Central and take full advantage of:}

- Convenient online submission

- Thorough peer review

- No space constraints or color figure charges

- Immediate publication on acceptance

- Inclusion in PubMed, CAS, Scopus and Google Scholar

- Research which is freely available for redistribution

Submit your manuscript at www.biomedcentral.com/submit 